\title{
Hispanismes
}

Revue de la Société des Hispanistes Français

$17 \mid 2021$

Murs, barrières, obstacles dans les mondes

hispaniques I

\section{Des lignes entre les murs, des murs entre les lignes}

La poésie carcérale de Marcos Ana

Líneas entre muros, muros entre líneas: la poesía carcelaria de Marcos Ana

Lines behind walls, walls between the lines : the prison poetry of Marcos Ana

Isabelle Pouzet Michel

\section{(2) OpenEdition}

\section{Journals}

Édition électronique

URL : https://journals.openedition.org/hispanismes/13608

DOI : 10.4000/hispanismes.13608

ISSN : 2270-0765

Éditeur

Société des Hispanistes Français

Référence électronique

Isabelle Pouzet Michel, «Des lignes entre les murs, des murs entre les lignes », Hispanismes [En ligne], 17 | 2021, mis en ligne le 01 juin 2021, consulté le 27 janvier 2022. URL : http://

journals.openedition.org/hispanismes/13608 ; DOI : https://doi.org/10.4000/hispanismes.13608

Ce document a été généré automatiquement le 27 janvier 2022.

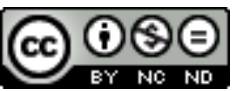

Les contenus de cette revue sont mis à disposition selon les termes de la Licence Creative Commons Attribution - Pas d'Utilisation Commerciale - Pas de Modification 4.0 International. 


\title{
Des lignes entre les murs, des murs entre les lignes
}

\author{
La poésie carcérale de Marcos Ana \\ Líneas entre muros, muros entre líneas: la poesía carcelaria de Marcos Ana \\ Lines behind walls, walls between the lines : the prison poetry of Marcos Ana
}

Isabelle Pouzet Michel

1 Unir le motif du mur à celui de la prison semble redondant tant l'emprisonnement ne peut être dissocié des murs, tant les murs sont la condition même de cet emprisonnement. Pourtant, quand il s'agit de poésie carcérale, l'allusion aux murs n'est pas systématique. Selon Aurore Ducellier, les références aux conditions de détention sont rares dans la poésie carcérale du premier franquisme qu'elle situe entre 1939 et $1945^{1}$. Cette absence peut surprendre mais elle s'explique par la censure que subissent les poèmes et donc par l'autocensure que finissent par s'imposer leurs auteurs craignant de voir leurs textes détruits. En revanche, dans la deuxième moitié de la période, qui correspond aux années 50 et 60 , la censure moins véhémente offre la possibilité à certains poètes, comme Marcos Ana qui a commencé à écrire en prison au milieu des années 50, d'écrire sur leurs conditions de vie en détention.

2 L'Espagnol Marcos Ana, de son vrai nom Fernando Macarro Castillo a été emprisonné pendant vingt-deux ans et sept mois. Il est arrêté en mars 1939, à l'âge de 19 ans; il est sorti de prison le 17 novembre 1961, à l'âge de 41 ans. Condamné à mort à deux reprises, sa peine est à chaque fois commuée en trente ans de prison, la peine maximale ${ }^{3}$, et il y serait resté enfermé jusqu'en 1980 s'il n'avait pas été libéré plus tôt. Après être passé par différents camps puis par différentes prisons espagnoles, la prison centrale de Burgos est finalement celle où il purge la plus grande partie de sa peine, loin de la ville d'Alcalá de Henares où vivait sa famille. Comme il le raconte dans son autobiographie, c'est dans ces geôles qu'il s'est initié à la poésie ${ }^{4}$ et qu'il a rédigé la presque totalité de son œuvre. Après sa libération, de nombreuses éditions de ses poèmes paraissent, mais il écrit peu, très occupé par ses responsabilités politiques et son engagement pour l'amnistie à travers le monde ${ }^{5}$. 
3 Manuel Aznar Soler définit la poésie de Marcos Ana comme une poésie de « cri, de lutte, d'accusation et d'espoir $»^{6}$. Effectivement, il s'agit d'une poésie composée dans l'urgence, tant les conditions de création sont rudimentaires : il écrit dans sa cellule, la nuit, à la lumière de la lampe à pétrole, souvent interrompu par les «alertas", les cris des sentinelles ${ }^{7}$. Le papier est rare à tel point que parfois il se voit obligé d'écrire sur d'autres supports tels que le papier à cigarettes ${ }^{8}$ et que la moindre feuille, la moindre marge doit être remplie de mots. Diverses éditions latino-américaines, dont la première au Mexique en 1959, portent sa poésie tout autant que sa cause à la connaissance du public $^{9}$. En Espagne, ses poèmes sont publiés pour la première fois en 1977 sous le titre Las soledades del muro puis, plus récemment en 2011, cinq ans avant sa mort, dans une édition augmentée titrée Poemas de la prisión y la vida. Il a également publié deux récits autobiographiques en Espagne dans les années 2000.

Qu'ils soient symboliques ou bien réels, les murs occupent toute la place dans ses poèmes de la prison, et à titre d'exemple, dans Poemas de la prisión y la vida ${ }^{10}$ le terme " muro » apparaît une ou plusieurs fois dans vingt poèmes distincts, c'est-à-dire plus de la moitié des textes réunis dans l'ouvrage. D'ailleurs quelques titres de ses recueils l'annoncent, tels Las soledades del muro (1977) ou Te llamo desde un muro (1961) : le poète parle du mur depuis le mur. Mais quels sont ces murs? Sont-ils seulement ceux de sa cellule ou ceux de la cour? Que signifient-ils? Quelle est leur portée symbolique dans son discours poétique?

\section{Des murs de la cellule à ceux du tombeau}

5 Lorsque María Teresa de León lui envoie un pull-over tricoté de ses mains et qu'elle glisse dans le paquet un message dans lequel elle lui demande de lui raconter sa vie en prison, Marcos Ana lui répond par ces quelques vers :

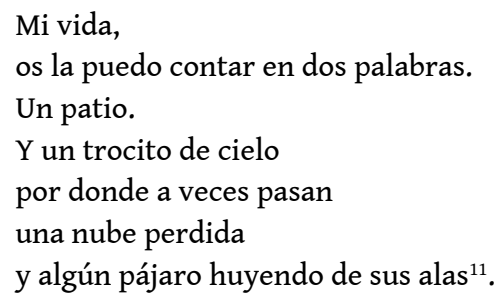

6 Au fil des jours monotones en prison, nul paysage ne s'offre au regard du détenu que celui des murs de la cour et de sa cellule. Ce «petit bout de ciel » aperçu depuis la cour ou entrevu par la lucarne depuis le fond de sa geôle ${ }^{12}$ constitue l'unique horizon pour le poète. Malgré la fréquence de leurs occurrences dans ses poèmes, «el muro » ou «los muros » (puisqu'il emploie aussi bien le pluriel que le singulier) ne désignent que les murs qui ceinturent les deux espaces où le prisonnier passe tout son temps : la cour, le jour et la cellule, la nuit. «Pared», nous le verrons, prend un autre sens sous la plume de Marcos Ana, tandis que d'autres murs, comme ceux des couloirs parcourus, ne sont pas mentionnés.

7 Dans le poème «La noche es mi refugio ", les murs de la cellule et ceux de la cour de promenade sont à la fois le lieu, le cadre et l'outil de l'enfermement :

Mares de sombra me rodean. Prietos

cinchos de alerta y muro.

(Ya la tarde 
como la puerta de celda se ha cerrado

contra la luz y el aire) ${ }^{13}$.

8 Mais bien vite, le mur va se parer de sens métaphoriques en venant désigner l'enfermement et la souffrance induite par cette contrainte. Tout d'abord, les murs blessent. Dans « Pequeña carta al mundo », les pierres de la cour sont coupantes :

$\mathrm{Y}$ en las piedras lacerantes

de este patio gris, desierto,

mi grito, como una estatua

crucificada y rota, en el centro ${ }^{14}$.

9 Ainsi sont les murs de la prison de Burgos, que Marcos Ana invoque dès le premier mot de « Prisión central », « muros »:

Muros hirsutos. Asperas cortezas

donde el hombre se duele cada día.

Apretada oquedad de llaga y fosa.

Socavón de Castilla. Lento espanto.

Catedral invertida hacia la tumba,

bajo una piel de piedra cancerosa.

Hay un árbol, aquí, pleno, enterrado,

de corazones vivos, que semejan

tréboles rojos en la luz borrosa:

muchas hojas, sin sangre, van cayendo;

mas su raíz fosfórica florece

una bandera abierta en cada losa.

$\mathrm{Y}$ en esta pena oscura donde habita

mi corazón en sombras, ya tan sólo

la luz de esa bandera es asombrosa ${ }^{15}$.

10 Composé de cinq tercets d'hendécasyllabes, « Prisión central » synthétise les trois sens donnés au mur dans la poésie de Marcos Ana : le mur blesse, tue, devient le tombeau du détenu. Le premier vers est abruptement coupé à l'hémistiche, où le point traduit la cruauté des murs, personnifiés par l'adjectif « hirsutos ». Le mur blesse, agresse, casse. Les échos phoniques du vers créés par les rimes internes « uros/utos » et «eras/ea » associées à l'allitération en « $\mathrm{s}$ » viennent souligner la violence de la cassure que le mur inflige au sujet poétique et qui s'incarne dans ce point surgi à l'hémistiche, mutilant le vers comme l'est l'âme du prisonnier ${ }^{16}$. Tandis que la brisure est associée aux murs, la douleur qui en découle n'a pas de limite : elle déborde du vers par l'enjambement et l'allitération en « $d$ » faisant résonner les corps des hommes meurtris par les parois acérées de leurs geôles.

11 Plus encore qu'une arme, le mur, métonymie de la prison, est assassin. Après avoir convoqué l'image de la blessure, le poète file aussitôt la métaphore du tombeau, fréquente dans la poésie carcérale de l'époque ${ }^{17}$. La verticalité structure le second tercet et représente cette descente inexorable et lente, ce «lento espanto ", vers le fond, vers la mort: "Catedral invertida hacia la tumba,/ bajo una piel de piedra cancerosa. " Au lieu d'élever vers les cieux divins comme le fait une cathédrale, cette construction humaine qu'est la prison devenue "catedral invertida " enfonce le prisonnier dans le sol en le conduisant tout droit à la mort. Le rapprochement paronomastique entre « piel » et « piedra » du dernier vers introduit un ultime sens : si le prisonnier perd sa vie dans ce tombeau qu'est la prison, en revanche celle-ci semble bel et bien animée, mais porteuse de menaces : «bajo una piel de piedra cancerosa ». 
12 Le poème « ¿La vida?» développe une image similaire lorsque le sujet poétique s'interroge sur la vie à l'extérieur, une vie dont il a fini par oublier les contours, les couleurs, les odeurs. Il a l'impression que la seule réalité qui existe est la sienne : « ¿O sólo queda esta fosa, /la luz de una sepultura/ y la canción de mis losas? ${ }^{18}$. La rime entre «fosa » et « losa », associant les dalles du sol et le trou, construit implicitement l'image de la pierre tombale.

Dans la poésie carcérale, la prison comme métaphore du tombeau est commune et ne naît évidemment pas sous la plume de Marcos Ana. Elle figure notamment dans les derniers poèmes de Miguel Hernández, pour qui Marcos Ana avait beaucoup d'admiration ${ }^{19}$. C'est le cas du poème "Sepultura de la imaginación » que Miguel Hernández a écrit en prison, quelques mois avant sa mort. Ce poème aux allures de fable et structuré par le motif de la verticalité, met en scène un maçon qui cherche à élever des murs. Les pierres sont aussi légères que des plumes, que l'espoir et l'optimisme qu'il associe à son œuvre: «piedras de plumas, muros de pájaros los mueve/ una imaginación al mediodía $»^{20}$. Mais, brutalement, la pierre retrouve sa pesanteur et devient l'agent de la construction précipitant le maçon dans sa geôle mortifère par un enjambement final : «Aquel hombre labraba su cárcel. Y en su obra/ fueron precipitados él y el viento $\aleph^{21}$. Miroir de sa propre situation, ce poème montre à quel point l'enfermement a fait perdre ses illusions à son auteur au point de le plonger dans un profond désespoir.

\section{Le corps se fait pierre}

14 À l'évocation des murs s'ajoute celle des pierres, omniprésentes, des dalles que foulent les prisonniers jusqu'aux hauts murs de la cour. Non seulement elles leur rappellent à chaque instant leur condition mais elles les transforment, les modèlent. La prison change l'homme en pierre, le pétrifie. Dans «Imaginaria ", le sujet poétique raconte sous la forme d'un témoignage qu'il a vu pleurer son co-détenu puis il insiste sur la souffrance que provoque cet enfermement représenté par les dalles, « las losas »: «Ya sabéis que a mí las losas/ me han gastado hasta los huesos/ del corazón,/ pero ver llorar a un hombre/ es algo, siempre, tremendo $»^{22}$. La pierre épuise les corps et les âmes.

Dans son célèbre poème « Mi corazón es patio ", le préféré de Rafael Alberti ${ }^{23}$ et versifié en arte menor ce qui en facilite la mémorisation, Marcos Ana insiste également sur la minéralité de l'univers carcéral. Ici, il troque le mots "muros " pour le mot "patio » qui n'en est pas moins douloureux : «Un patio bajo un cielo de fosa, desgarrado,/ que acuchillan y acotan/ muros y pararrayos $»^{24}$. Comme l'exprime le titre, le monde du sujet poétique se réduit à la cour. Le mouvement circulaire des prisonniers en promenade est repris par la circularité du texte, qui se termine comme il a commencé, ainsi que par l'anaphore, la répétition de tournures syntaxiques proches et la reprise d'une strophe à la manière d'un refrain. Le poème tourne en rond, à l'instar de ses dernières strophes et des prisonniers en promenade :

[...] Amanezco, y ya todo

-fuera del sueño-

es patio:

un patio donde giran

los hombres sin espacio. 


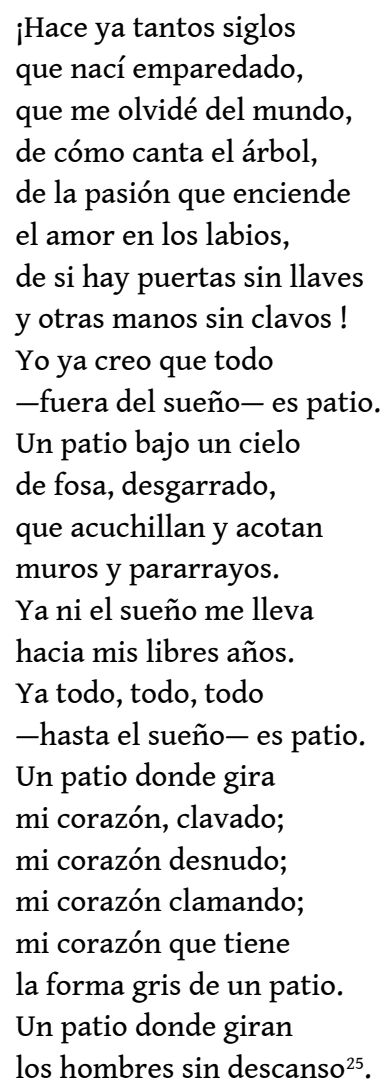

La pensée sans issue est à l'image du rêve qui ne semble offrir d'autre perspective que cet éternel et immuable décor de pierre. Dans une interview donnée en 1976, Marcos Ana raconte qu'à la fin de son emprisonnement, il avait passé tellement de temps en prison qu'il ne rêvait plus que d'elle ${ }^{26}$. Dès lors la pierre, le mur font partie de son identité, la voix poétique dit d'ailleurs dans ce même poème qu'elle est née emmurée : « nací emparedado ${ }^{27}$.

\section{3. Écrire sur les murs}

Indissociable de l'enfermement, le mur est également investi d'un tout autre rôle lorsqu'il devient le support même de l'écriture. Marcos Ana raconte dans son autobiographie que les condamnés à mort laissaient une trace écrite de leur passage la nuit de leur « saca ", lorsque leurs geôliers venaient les chercher pour les fusiller. Ces petits mots souvent adressés à la famille sont les « notas de capilla » ${ }^{28}$ que le condamné laissait dans sa cellule. Ils les écrivaient sur du papier ou, si celui-ci faisait défaut, sur les murs. Un autre poète, Manuel de la Escalera, ami de Marcos Ana dont il fait la connaissance dans la prison de Burgos et qui comme lui a été emprisonné pendant plus de vingt ans, a raconté dans le journal rédigé alors qu'il était condamné à mort à Alcalá de Henares de quelle manière les murs des cellules servaient de palimpsestes :

A derecha e izquierda de nuestra celda hay otros condenados que esperan una muerte más próxima, pues llevan más tiempo que nosotros. Además, en nuestras paredes quedan inscripciones, algunas muy antiguas y borrosas, otras recientes, en las que nos hablan otros que pasaron por aquí, nuestros antecesores. Algunas de estas inscripciones son de una simplicidad conmovedora. Se esfuerzan en registrar con rigor notarial el día y la hora en que entraron a la celda con la condena de muerte, dando su nombre y apellidos, el nombre del pueblo donde nacieron, o, en 
otras, la hora, día, nombre, apellidos y pueblo de los sacados, de aquellos que vieron salir a la muerte, estando allí, siendo testigos. ¿A quiénes se dirigen estas líneas? Sin duda a nosotros, a los que venimos detrás. En una u otra forma, a la posteridad, aun cuando sea en su forma más rudimentaria ${ }^{29}$.

Si la plupart du temps, les traces que laissaient les condamnés sur les murs de leur cellule se limitaient à des indications biographiques ou temporelles, il pouvait arriver que certains y griffonnent quelques vers. Manuel de la Escalera évoque le cas de Francisco Burgos Lecea qui avait écrit un poème sur les murs de son cachot alors qu'il se trouvait enfermé à la Puerta del Sol ${ }^{30}$.

Marcos Ana, condamné à mort à deux reprises, aurait dans ces circonstances laissé lui aussi sa trace sur les murs de sa cellule. Une première fois en 1944, dans la prison d'Ocaña, il aurait écrit au charbon un poème que les gardiens ont effacé ; et une seconde fois lorsqu'il apprit, après une nuit passée à attendre "la saca ", que sa peine était commuée ${ }^{31}$. Comme le rappelle Aurore Ducellier, «le poème-graffiti » est une pratique répandue en prison qui n'est évidemment pas propre à Marcos Ana et qui lui a cependant permis de "s'épancher, de canaliser et de sublimer une émotion, mais aussi, peut-être, de laisser volontairement un message d'espoir à ses successeurs dans l'antichambre de la mort incertaine $~^{32}$.

Le poète fait allusion à cette pratique dans le romance « Pequeña carta al mundo ». Dans celui-ci, le sujet poétique s'adresse à des lecteurs imaginaires, hors les murs. Il établit une nette distinction entre le mot "muro" connoté négativement car associé à la prison et le mot «pared » qui transmet un message d'espoir. Le terme ne désigne pas les murs de la prison mais ceux où les prisonniers laisseront leurs noms, leur trace même après leur mort; ce sont ceux grâce à qui leurs voix survivront, lorsqu'ils seront passés de vie à trépas :

[...] Si llegáis ya tarde un día y encontráis frío mi cuerpo, de nieve a mis camaradas entre sus cadenas muertos... recoged nuestras banderas, nuestro dolor, nuestro sueño, los nombres que en las paredes con dulce amor grabaremos.

Y si no cerráis los ojos ¡dejadnos los muros dentro!, que se pudran con el polvo de nuestra carne no puedan ser nuevas tumbas de presos. No sabéis lo que es un hombre, sangrando y roto, en un cepo.

Si lo supieseis vendríais en las olas y en el viento, desde todos los confines, para salvar lo que es vuestro. Si llegáis ya tarde un día y encontráis frío mi cuerpo, buscad en las soledades del muro mi testamento: al mundo le dejo todo lo que tengo y lo que siento, lo que he sido entre los míos, lo que soy, lo que sostengo: una bandera sin llanto, 
un amor, algunos versos...

y en piedras lacerantes

de este patio gris, desierto,

mi grito, como una estatua

terrible y roja, en el centro ${ }^{33}$. hommes, les enferment, les oppressent jusqu'à parfois les mener à la mort, ne sont pas infranchissables. La revue Muro en est la preuve et un exemple parmi tant d'autres. Ces murs n'empêchent pas les messages de passer, dans un sens, du dehors jusqu'à la cellule ou dans l'autre, de la cellule jusqu'à l'extérieur. Dans la revue Muro est par exemple reproduite en fac-similé une lettre, que la fille d'un prisonnier a adressée à son père, un paysan andalou. Marcos Ana raconte dans son autobiographie, Decidme cómo es un árbol, comment à Burgos les prisonniers parvenaient à faire pénétrer et à diffuser des livres de poètes contemporains en collant les pages de leurs œuvres sous les couvertures des livres de la bibliothèque de la prison ${ }^{37}$. Marcos Ana avait d'ailleurs déjà publié certains de ses poèmes en Amérique latine avant sa sortie de prison en les faisant passer à l'extérieur de façon clandestine : en 1959, il publie au Mexique Te llamo desde un muro avec l'aide de Juan Rejano ; en 1960, paraissent en Argentine le recueil Poemas desde la cárcel et sept poèmes, à l'occasion de la seconde conférence latinoaméricaine pour l'amnistie pour les prisonniers et les exilés politiques d'Espagne et du Portugal, célébrée à Buenos Aires.

Les prisonniers pouvaient faire preuve d'une grande ingéniosité pour cacher leurs textes dans leur cellule, tel le je poétique de «La noche es mi refugio » racontant qu'il dissimule ses vers dans ses souliers: «Después cuando amanezcan/ los ojos y las llaves,/ me guardaré la voz en un zapato/y aromarán las losas mi mensaje ${ }^{38}$. 
$\mathrm{Au}$ terme de cette réflexion menée à propos des murs dans la poésie de Marcos Ana, le lecteur pourrait croire que sa poétique est empreinte de désespoir. Il n'en est rien, car en réalité il jaillit de ses vers un puissant souffle de vie. C'est sûrement l'une des raisons qui lui a valu son succès après sa libération, assortie au fait que ses textes, souvent en vers d'arte menor et aux rimes faciles, sont très accessibles. Lui-même, malgré tous les tourments que lui a causés son emprisonnement, tels que la torture ${ }^{39}$, l'isolement ${ }^{40}$, la maladie $^{41}$ ou encore la perte de sa mère, n'a jamais sombré dans un fatal désespoir ${ }^{42}$; au contraire, il a conservé une sorte de force vitale, peut-être liée à son jeune âge et au désir de vivre la vie qui l'attendait dehors.

Le poème "Prisión central » évoqué plus haut file tout d'abord la métaphore du tombeau associé à la verticalité ; ensuite, convoquant l'arbre, symbole des prisonniers et, par extension de leur lutte, il contrecarre le mouvement descendant et mortifère de la prison:

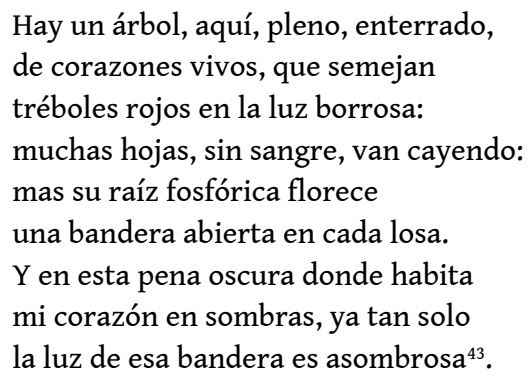

Même si l'arbre est enterré, ses racines font palpiter la vie sous les dalles qu'elles illuminent. Le rythme du vers, qui s'accélère brusquement sous l'impulsion d'un contraccent «mas su raíz fosfórica florece» et de l'allitération, traduit cette vie qui frémit sous la pierre. On ne peut s'empêcher de penser à la description que fait non sans humour Manuel de la Escalera dans Muerte después de Reyes, des arbres de la cour de la prison d'Alcalá de Henares qui ont grandi tordus pour chercher la lumière, comme pour défier la loi des geôliers ${ }^{44}$. Chez Marcos Ana, l'arbre est certes métaphorique puisqu'il est associé au combat politique, mais le symbole reste le même: l'enfermement ne peut rien contre la vie. À l'inverse, la pierre peut parfois être un symbole de courage et de lutte comme dans « Norma » où les pierres donnent vie, au sens propre comme au sens figuré, au squelette du poème : «Quiero que mis poemas tengan hueso/ y estructura de piedras palpitantes $»^{45}$. Ainsi, les vers de Marcos Ana fissurent les murs de la prison créant autant de brèches d'où s'échappe un souffle de vie nourri par un indéfectible espoir.

\section{BIBLIOGRAPHIE}

Juan José del ÁGUILA TORRES, « № 76: La Causa № 120.967 contra Marcos Ana y otros cuatro presos, su segundo consejo de guerra y la condena de 20 de diciembre de 1943 », blog Justicia y dictadura [consulté le 23 janvier 2021], <URL: https://justiciaydictadura.com/la-causa-no-120-967-contra- 
marcos-ana-y-otros-cuatro-presos-su-segundo-consejo-de-guerra-y-la-condena-de-20-dediciembre-de-1943/>.

Marcos ANA, « Cartas a nuestros amigos de América latina », Muro. Páginas de la prisión, août 1961, Organización para la Amnistía General en España y Portugal, août 1963.

Marcos ANA, «Cultura entre rejas », Cultura de la República. Revista de Análisis Crítico (CRRAC), nº 1 (2017) [consulté le 22 février 2021] <URL: https://revistas.uam.es/crepublica/issue/view/672/380 $>$.

Marcos ANA, Decidme cómo es un árbol, Barcelona, Umbriel editores, 2007.

Marcos ANA, Las soledades del muro, illustrations de Agustín Ibarrola, Madrid, Akal editor, 1977.

Marcos ANA, « María Teresa León, una mujer comprometida con su tiempo », Homenaje a María Teresa León, Madrid, Cursos de Verano de la Universidad Complutense de Madrid en El Escorial-1989, 1990, p. 41-50.

Marcos ANA, Poemas de la prisión y la vida, Barcelona, Umbriel editores, 2011.

Marcos ANA, Sino sangriento. Homenaje a voz apagada para Miguel Hernández, édition non vénale, Madrid, 2007.

Marcos ANA, Te llamo desde un muro. Poemas de la prisión, Buenos Aires, Organización para la Amnistía General en España y Portugal, 1961.

Manuel AZNAR SOLER, « Marcos Ana, un poeta en el penal de Burgos », communication prononcée au congrès Los campos de concentración y el mundo penitenciario en España durante la guerra civil y el franquismo organisé par le Centre d'Études sur les Époques Franquiste et Démocratique, Université Autonome de Barcelone, 21, 22 et 23 octobre 2002, p. 445-467 [consulté le 12 février 2021] <URL: https://silo.tips/download/marcos-ana-un-poeta-en-el-penal-de-burgos > .

Aurore DUCELLIER, Les voix résilientes. La poésie carcérale sous le premier franquisme, t.1, thèse de doctorat, Université Paris Ouest Nanterre, non publiée, soutenue en juin 2016.

Manuel de la ESCALERA, Muerte después de Reyes, Madrid, Akal editor, 2015.

Miguel HERNÁNDEZ, Obra completa. Poesía I, édition critique d'Agustín Sánchez Vidal et José Carlos Rovira, Madrid, Espasa-Calpe, 1992.

Muro. Páginas de la prisión, agosto de 1961, Organización para la Amnistía General en España y Portugal, agosto de 1963.

\section{NOTES}

1. Aurore DUCELLIER, Les voix résilientes. La poésie carcérale sous le premier franquisme, t.1, thèse de doctorat, Université Paris Ouest Nanterre, non publiée, soutenue en juin 2016, p. 579-588. Elle observe également que le style de Marcos Ana est plus pamphlétaire que celui d'autres poètes prisonniers de la même époque, ce qui peut expliquer aussi la présence plus forte de ces références : Aurore DUCELLIER, ibid., p. 234.

2. Il a choisi ce nom de plume en hommage à ses parents. Dans son autobiographie Decidme cómo es un árbol, il raconte que son père, Marcos, est mort victime dans un bombardement de l'aviation allemande en 1937 et que sa mère, Ana, est décédée alors qu'il était encore prisonnier : Marcos ANA, Decidme cómo es un árbol, Barcelona, Umbriel editores, 2007, p. 48-49 et p. 156.

3. Marcos Ana a été condamné une première fois à la peine de mort en 1941 mais sa peine a été commuée car il était mineur lors des faits qui lui ont été reprochés. La seconde fois, il a été jugé 
et condamné à mort pour avoir élaboré une revue clandestine Juventud des JSU (Juventudes Socialistas Unificadas) en prison en 1943. Sur les détails des jugements de Marcos Ana, voir l'article de Juan José del AGUILA TORRES : « № 76: La Causa № 120.967 contra Marcos Ana y otros cuatro presos, su segundo consejo de guerra y la condena de 20 de diciembre de 1943 » sur son blog Justicia y dictadura https://justiciaydictadura.com/la-causa-no-120-967-contra-marcos-anay-otros-cuatro-presos-su-segundo-consejo-de-guerra-y-la-condena-de-20-de-diciembrede-1943/, consulté le 23 janvier 2021.

4. Marcos ANA, Decidme cómo es un árbol, op. cit., p. 167.

5. À José Martí Gómez et Josep Ramoneda, il explique aussi qu'après sa libération, il a souffert de claustrophobie et qu'il lui était impossible de rester enfermé pour écrire : José MARTI GOMEZ et Josep RAMONEDA, « Marcos Ana el decano », Por favor, $1^{\mathrm{er}}$ mars 1976. L'interview a été publiée dans Marcos Ana, Las soledades del muro, illustrations de Agustín Ibarrola, Madrid, Akal editor, 1977, p. 69-83.

6. Manuel AZNAR SOLER, "Marcos Ana, un poeta en el penal de Burgos", communication prononcée au congrès «Los campos de concentración y el mundo penitenciario en España durante la guerre civil y el franquismo» organisé par le Centre d'Etudes sur les Epoques Franquiste et Démocratique, Université Autonome de Barcelone, 21, 22 et 23 octobre 2002, p. 451, consulté le 12 février 2021. Nous traduisons.

7. Marcos ANA, Las soledades del muro, op. cit., p. 8 : «Es una poesía urgente por su contenido y porque tenía que ser desgarrada sobre el papel, mientras en el silencio cuadrado y terrible de la cárcel se escuchaban los pasos de los carceleros y los alertas de los centinelas que vigilaban desde el recinto ».

8. Marcos ANA, Decidme cómo es un árbol, op. cit., p. 174.

9. Il publie au Mexique Te llamo desde un muro. Poemas de la prisión chez España popular avec l'aide de Juan Rejano. Ce même recueil est publié en 1961 en Argentine, en 1963 au Venezuela. En 1960 paraît Poemas desde la cárcel en Uruguay ainsi qu'au Brésil.

10. Marcos ANA, Poemas de la prisión y la vida, Barcelona, Umbriel editores, 2011.

11. Marcos ANA, Decidme cómo es un árbol, op. cit., p. 173-174. Il a écrit ces vers sur du papier à cigarettes qu'il parviendra à faire sortir de prison en les dissimulant dans un tube de dentifrice.

12. Marcos ANA, ibid., p. 158-159. Marcos Ana raconte que dans la prison d'Alcalá de Henares il passait des heures à regarder le ciel nocturne depuis la lucarne de sa cellule ce qui lui a valu d'être sanctionné.

13. Marcos ANA, Las soledades del muro, op. cit., p. 33.

14. Marcos ANA, Poemas de la prisión y la vida, op. cit., p. 18.

15. Marcos ANA, Las soledades del muro, op. cit., p. 15.

16. Cette ponctuation nous rappelle les vers mutilés de Juan Gelman qui, pour traduire les douleurs de l'exil et de la perte du fils, a usé de la barre oblique dans ses poèmes Interrupciones 2. Il est regrettable que Marcos Ana n'en ait pas fait plus usage.

17. Aurore DUCELLIER, Les voix résilientes. La poésie carcérale sous le premier franquisme, op. cit., p. 234-235.

18. Marcos ANA, Las soledades del muro, op. cit., p. 17-18.

19. Marcos ANA a pu lire l'œuvre publiée de Miguel Hernández en prison comme il le raconte dans Decidme cómo es un árbol, op. cit., p. 179-181. On ignore cependant s'il connaissait «Sepultura de la imaginación ». Il lui a rendu hommage à l'automne 1960 avec la pièce de théâtre Sino sangriento. Homenaje a voz ahogada para Miguel Hernández écrite avec d'autres prisonniers de Burgos. Celle-ci a été publiée dans une édition limitée et non commercialisable en 2007.

20. Miguel hernandez, Obra completa. Poesía I, édition critique d'Agustín Sánchez Vidal et José Carlos Rovira, Madrid, Espasa-Calpe, 1992, p. 758.

21. Id. 
22. Marcos ANA, Poemas de la prisión y la vida, op. cit., p. 44.

23. Marcos ANA, Decidme cómo es un árbol, op. cit., p. 177.

24. Marcos ANA, Las soledades del muro, op. cit., p. 21.

25. Ibid., p. 21-22.

26. José mARTI GOMEZ et Josep RAmonedA, « Marcos Ana, el decano », Por Favor, 1 mars 1976, publié dans Marcos ANA, Las soledades del muro, op. cit., p. 72.

27. On retrouve cette réflexion chez Manuel de la Escalera qui a rédigé entre décembre 1944 et janvier 1945 un journal alors qu'il était incarcéré et condamné à mort. Dans le passage de son journal Muerte después de Reyes publié pour la première fois au Mexique en 1966, il emploie lui aussi le terme "emparedado" lorsqu'il décrit la situation des prisonniers enfermés dans des cachots au sous-sol de la Direction générale de sécurité à Madrid, à la Puerta del Sol qui perçoivent au-dessus de leurs têtes la bruyante rumeur de la vie urbaine : «Los calabozos de la Puerta del Sol están esperando el Wilde que escriba su balada. Yo, entre otros deseos no logrados, me llevaré a la tumba este de ser Virgilio de su infierno, o, simplemente, el reportero dantesco que lo describa con amplitud, pues haría falta otro libro. En él contaría cómo oyen los soterrados, los emparedados, nuevos tántalos, los ecos y rumores de la vida y del tráfico en el centro de una gran ciudad ». Nous soulignons: Manuel de la ESCALERA, Muerte después de Reyes, Madrid, Akal editor, 2015, p. 57.

28. Marcos ANA, Decidme cómo es un árbol, op. cit., p. 100-105.

29. Manuel de la ESCALERA, Muerte después de Reyes, op. cit., p. 15.

30. Ibid., p. 114.

31. Aurore DUCELLIER, Les voix résilientes. La poésie carcérale sous le premier franquisme, op. cit., p. 190-191. Le premier poème est le suivant : «Jamás horada mi alma,/ jamás ciega mi vida/ una celda sin ventana./ Solo con mi dolor y mi condena,/ Sin ver que nuestros gritos arden bosques,/ Sin escuchar que el fuego nos contesta,/ Y nos llaman cien pueblos que nos buscan,/ Con sus lámparas rojas avanzando,/ Desde las cinco partes de la Tierra.» Le second est celui-ci : «Dos veces vino la muerte,/ Y dos se fue arrepentida./ Dicen que marchó ofendida/ porque no doblé mi frente./ ¡Por eso dejó mi vida! ».

32. Ibid., p. 191.

33. Marcos Ana, Las soledades del muro, op. cit., p. 35-36. Nous soulignons.

34. Marcos ANA, "Cartas a nuestros amigos de América latina », Muro. Páginas de la prisión, août 1961, Organización para la Amnistía General en España y Portugal, août 1963.

35. Marcos ANA, « María Teresa León, una mujer comprometida con su tiempo », Homenaje a María Teresa León, Madrid, Cursos de Verano de la Universidad Complutense de Madrid en El Escorial-1989, 1990, p. 44.

36. Marcos ANA, "Cartas a nuestros amigos de América latina », Muro. Páginas de la prisión, op. cit., sans pagination. On retrouve un récit semblable dans le journal de Manuel de la Escalera publié sous le titre Muerte después de Reyes. Alors qu'il était condamné à mort à la prison d'Alcalá de Henares, il raconte que les bruits de bottes, les claquements des portes, le crissement des pneus des voitures dans la cour et enfin les cris déchirants des prisonniers emportés par les gardiens étaient autant d'indices des exécutions qui auraient lieu à l'aube : Manuel de la ESCALERA, Muerte después de Reyes, op. cit., p. 105-109.

37. Marcos ANA, Decidme cómo es un árbol, op. cit., p. 173.

38. Marcos ANA, Las soledades del muro, op. cit., p. 34.

39. Marcos ANA, Decidme cómo es un árbol, op. cit., p. 119.

40. Ibid., p. 167.

41. Ibid., p. 75-84 et p. 166-167. Marcos Ana a été gravement malade à la prison de Porlier et a vécu deux ans à l'infirmerie de la prison centrale de Burgos. 
42. Ibid., p. 151. Il a fait une tentative de suicide dans le but d'entrer en contact avec un autre prisonnier.

43. Marcos ANA, Las soledades del muro, op. cit., p. 15.

44. Manuel de la ESCALERA, Muerte después de Reyes, op. cit., p. 75-76: « A más del patio pequeño, al que salimos los condenados a muerte, hay otro patio grande, del que ya hablé, con dos acacias y varios pinos. Éstos, que nacieron torcidos por haber tenido que luchar contra el viento de otros siglos, acaso en los tiempos de Cervantes, cuando no estaban protegidos por estos muros encarcelados-, ahora que lo están, como presos de verdad, no han podido rectificar sus torcidas inclinaciones. Una buena lección para los señores penalistas. »

45. Marcos ANA, Las soledades del muro, op. cit., p. 39.

\section{RÉSUMÉS}

Le poète espagnol Fernando Macarro Castillo (1920-2016), connu sous le nom de plume Marcos Ana, détient le triste record du nombre d'années passées dans les prisons franquistes, puisqu'il a été incarcéré vingt-deux ans et sept mois, de 1939 à 1961. Libéré alors qu'il avait à peine une quarantaine d'années, il a dû réapprendre à vivre et a consacré sa vie à défendre la cause des prisonniers politiques à travers le monde. Mettant en regard les témoignages de Marcos Ana avec ses poèmes rédigés en prison, nous saisirons les traces qu'ont laissées ces nombreuses années d'emprisonnement sur son écriture et nous verrons à quel point le motif du mur joue un rôle fondamental dans sa poésie.

Spanish poet Fernando Macarro Castillo (1920-2016), best known by his pen name 'Marcos Ana', holds the sad record of the longest term served in Francoist prisons - as he spent twenty-two years and seven months in gaol, from 1939 to 1961 . He was released in his early forties and had to get to grips with real life again. He then devoted the rest of his life to defending political prisoners throughout the world. We shall compare the poems Marcos Ana wrote in gaol with his testimonies, in order to trace back the influence of his long prison term on his writing, and establish to what extent the motif of the wall plays a fundamental role in his poetry.

\section{INDEX}

Mots-clés : poésie, Marcos Ana, mur, prison

Keywords : poetry, Marcos Ana, wall, prison

\section{AUTEUR}

\section{ISABELLE POUZET MICHEL}

Université Littoral Côte d'Opale 